\title{
Dalla demolizione del passato marxista al nuevo Chile. Propaganda e "guerra psicologica" nel primo anno di governo della dittatura di Augusto Pinochet
} \author{
war" during the first year of government of the dictatorship of Augusto Pinochet \\ ALESSANDRO GUIDA \\ Università degli Studi di Napoli "L'Orientale" (Italia) \\ https://orcid.org/0000-0002-8102-4754 \\ alessandroguida2@gmail.com
}

From the demolition of the Marxist past to the nuevo Chile. Propaganda and "psychological

\section{SinOSSI}

La dittatura di Augusto Pinochet non governò esclusivamente attraverso la violenza fisica. Sul "fronte interno" la popolazione venne "conquistata" anche attraverso una manipolazione che fu implacabile e che venne condotta attraverso tutti i mezzi disponibili. In una fase iniziale, la propaganda e la "guerra psicologica" del regime ebbero a che vedere con i "guasti" prodotti dal governo Allende e con l'antimarxismo in generale. Parallelamente, prevalsero il richiamo a valori quali l'unità della nazione, la moralità, la necessità di ripristinare virtù e simboli tradizionali, nonché il tentativo di compattare la popolazione intorno agli obiettivi nazionali della Giunta. Il tutto si produsse nel quadro di una versione specifica e originale della "Dottrina della sicurezza nazionale", che iniziò a operare sin dalle prime battute della dittatura. Verso la metà del 1974 iniziò a manifestarsi la necessità di un cambio di rotta nella strategia comunicativa, che rispondeva a ragioni sia di ordine interno che esterno.

Parole chiave: Dittatura cilena; Dottrina della sicurezza nazionale; propaganda; guerra psicologica; "nuovo Cile".

\section{Abstract}

The dictatorship of Augusto Pinochet did not rule exclusively through physical violence. On the "internal front" the population was "conquered" also through a manipulation that was relentless and that was conducted through all available means. At an early stage, the regime's propaganda and "psychological warfare" concerned the "breakdowns" produced by the Allende government and the anti-Marxism in general. At the same time, the reference to values such as the unity of the nation, morality, the need to restore traditional virtues and symbols, as well as the attempt to compact the population around the national objectives of the Junta prevailed. All this took place within 
the framework of a specific and original version of the "National security doctrine", which began operating from the very beginning of the dictatorship. In the middle of 1974 the need for a change of direction in the communication strategy began to emerge, and it responded to both internal and external reasons.

Keywords: Chilean dictatorship; National security doctrine; propaganda; psychological warfare; "new Chile".

\section{11 SETTEMBRE 1974: UN ANNO DOPO IL GOLPE}

Secondo quanto riportato dalla stampa dell'epoca, le celebrazioni dell'11 settembre per commemorare il primo anno dal «pronunciamiento militar» ${ }^{1}$ erano state avviate già alle nove del mattino, con una cerimonia religiosa all'interno del Patio Alpatacal della scuola militare di Santiago, alla quale avevano assistito i quattro membri della Giunta di governo, gli stessi che trecentosessantacinque giorni prima «habían unido su decisión, su esfuerzo y dolorosos sacríficios de sangre de sus respectivos institutos para poner fin a los casi tres años de la UP y de Allende» ${ }^{2}$. Verso mezzogiorno, poi, mentre una folla di decine di migliaia di persone - almeno in base a quello che raccontavano i giornali - aveva incominciato ad occupare con "grande entusiasmo" il parco Bustamante, al fine di manifestare il proprio "commovente sostegno"3 all'esecutivo militare, il generale Augusto Pinochet Ugarte aveva dato inizio al suo discorso nella sala de Plenarios del vicino Edificio Diego Portales ${ }^{4}$, nel mezzo degli applausi e subito dopo che le note dell'inno nazionale avevano cessato di risuonare ${ }^{5}$.

Nessun successo politico, nell'intera storia della nazione, affermò in quella circostanza il presidente della Giunta e capo dello Stato, aveva avuto l'importanza degli eventi dell'11 settembre 1973. Appena un anno prima, a quella stessa ora, le Forze Armate

1 Il termine pronunciamiento nella tradizione spagnola e, successivamente, nel contesto latinoamericano, non era stato utilizzato semplicemente come sinonimo di "colpo militare", ma era stato frequentemente adoperato per indicare la legittimità dell'intervento dei militari - se non, addirittura, la sua obbligatorietà - in caso di minaccia all'interesse nazionale o a fronte di drammatiche crisi economiche, politiche o sociali; e questo, in virtù dell'obbligo, costituzionale e statutario, delle Forze Armate di difendere lo stato di diritto e di prevenire un'usurpazione di autorità (Loveman, 1999: XXVI). Un concetto che sottintende anche un intervento limitato, di breve periodo, da parte dei militari, finalizzato a ripristinare l'ordine per poi riconsegnare il potere nelle mani delle forze "democratiche".

2 Qué Pasa, Santiago: "La fiesta del once", 17/09/1974, p. 12.

3 El Mercurio, Santiago: "Estremecedor Respaldo", 12/09/1974, pp. 1-2.

4 L'edificio in questione era stato realizzato a tempo di record dal governo Allende per ospitare i lavori della III Conferenza delle Nazioni Unite sul commercio e lo sviluppo (Santiago, 13 aprile - 21 maggio 1972). Al termine di questa, era stato affidato al ministero dell'Educazione che lo aveva adibito a centro culturale (Centro Cultural Metropolitano Gabriela Mistral). Immediatamente dopo il golpe, il centro, ribattezzato con il nome del "padre della patria", Diego Portales, iniziò ad essere utilizzato come quartier general del regime, anche alla luce del fatto che il palazzo presidenziale de La Moneda era stato gravemente danneggiato nel corso delle operazioni militari dell' 11 settembre.

5 Las Últimas Noticias, Santiago: "Lágrimas arranco discurso", 12/09/1974, p. 2. 
avevano ripudiato il regime totalitario marxista-leninista e garantito la liberazione del paese. Non sarebbe mai stato ricordato a sufficienza lo stato di violenza materiale e spirituale, di corruzione amministrativa, di caos economico, di rottura della convivenza e delle istituzioni democratiche prodottosi durante la precedente amministrazione; anzi, la devastazione era stata tale - proseguì il dittatore - da rendere impossibile un semplice ritorno al passato: occorreva «recuperar a Chile como nación que estructura sus objetivos para proyectarse a mejores destinos como un Estado libre y soberano» ${ }^{6}$.

Durante quei dodici mesi, infatti, il Cile aveva visto sì una normalizzazione di tutte le proprie attività, ma, per via della vastità e della profondità dei danni prodotti dalla precedente esperienza, appunto, si era trattato di «un periodo de ajustes y ordenamento», più che di «un año de construcción». Eppure il mondo libero era invitato a meditare sull'esperienza di questo paese, perché i suoi insegnamenti oltrepassavano di gran lunga i suoi confini, dichiarava Pinochet. Nonostante il permanente attacco proveniente dall'esterno, il Cile avanzava, infatti, in modo sicuro e senza vacillare, e lo faceva sulla base di una Dichiarazione di princìpi sottoscritta dai membri della Giunta di governo appena sei mesi dopo la presa del potere ${ }^{7}$ e che lo aveva dotato di una solidità di concetti che poche nazioni al mondo potevano vantare. Su queste fondamenta, peraltro, una commissione di giuristi indipendenti stava preparando con cura il progetto di una nuova Costituzione, che avrebbe permesso al Cile di godere in futuro «de una democracia estable, ágil y moderna». La ristrutturazione totale del Gabinetto, avvenuta due mesi prima, non aveva rappresentato, infatti, un mero ricambio di ministri, ma aveva costituito l'inizio della "fase due" della pianificazione del governo, quella del consolidamento, una tappa, peraltro, proiettata verso una più stretta unione tra le Forze Armate e i civili ed una partecipazione crescente di questi ultimi nell'esecutivo. Superate le esigenze del primo anno «de la gran depuración», il passo successivo sarebbe stato, infatti, quello di procedere all'integrazione «entre los uniformados y la civilidad», nel comune compito di edificare «un Chile grande, libre y poderoso» ${ }^{8}$.

Il discorso del jefe de la nación non era durato tantissimo, riportava la stampa, eppure dalle sue parole erano emersi degli aspetti tutt'altro che di poco conto. Fra questi, in particolare, il fatto che il regime, attraverso il suo massimo esponente, aveva dato l'impressione di non limitarsi più a definire sé stesso per mera contrapposizione nei confronti del passato (marxista). Portata a termine la missione di "normalizzare" e "depurare" la nazione, esso prometteva adesso ai cileni qualcosa d'altro, un nuovo Cile, un futuro di grandezza.

Tutto questo emergeva, del resto, anche dalle pubblicazioni propagandistiche realizzate per l'occasione - ben 12 libri, annunciò il quotidiano El Mercurio $^{9}$-, fra cui il

$6 \quad$ El Mercurio: "Estremecedor Respaldo", cit., p. 2.

7 La Declaración de principios del marzo del 1974 fu il primo documento che definì le basi ideologiche ed i contenuti programmatici del nuovo regime.

8 El Mercurio: "Estremecedor Respaldo", cit., p. 2.

9 El Mercurio: "Un año de construcción”, 12/09/1974, pp. 21-23. 
testo Un año de construcción, in cui tutte le varie questioni (economiche, sociali, istituzionali) venivano analizzate sia sotto il profilo dello status quo che dal punto di vista della «planificación futura a medio y largo plazo» (Pinochet Ugarte et al., 1974) ${ }^{10}$, e, soprattutto, República de Chile 1974. Primer año de la reconstrucción nacional (Junta De Gobierno De Las Fuerza Armadas Y Carabineros De Chile, 1974). Quest'ultima pubblicazione, in particolare, non si limitò a reiterare le ragioni del rovesciamento di Allende, ma argomentò, anche attraverso le sue illustrazioni, le metafore del nuovo regime «por medio de los objetos y distintivos protocolares», allo scopo, non solo di informare, celebrare e giustificare, ma anche di «instruir, en su sentido literal», presentando «por primera vez, las diversas dimensiones del nuevo Estado: su estructura administrativa, sus pretensiones geopolíticas, su equipamiento y potencia militar, sus estandartes favoritos, sus edificios, su ceremonial y objetos, sus personajes populares» (Jara Hinojosa, 2011: 157-158). Attraverso un uso "accorto" delle immagini, e mettendo insieme alcuni dei più importanti atti emanati dall'esecutivo militare nel corso dei suoi primi 12 mesi di gestione, nonché alcuni dei principali discorsi tenuti dai membri della Giunta, l'intento era quello di presentare un contesto di assoluta normalità, all'interno del quale il nuovo governo, forte di una base dottrinaria solida e del sostegno della popolazione, stava dando vita ad un «nuevo régimen institucional y político, enraizado en la más pura tradición nacional, pero simultáneamente moderno, estable y creador» (Junta De Gobierno De Las Fuerza Armadas Y Carabineros De Chile, 1974: 5).

\section{EXTIRPAR EL CÁNCER MARXISTA HASTA LAS ÚLTIMAS CONSECUENCIAS (EN EL NOMBRE DE LA SEGURIDAD NACIONAL)}

Quando, l'11 settembre del 1973, i militari rovesciarono il governo di Salvador Allende, realizzarono il colpo di Stato con grande efficacia, non incontrando quasi alcuna forma di resistenza (Huneeus, 2016: 114-115). In pratica, l'operazione ebbe tutte le caratteristiche di un'invasione militare, con la Giunta che diresse un esercito di occupazione all'interno del suo stesso paese (Garretón et al., 1998: 11). Se il controllo militare del paese avvenne in maniera estremamente rapida, ugualmente veloce fu la definizione dei principali orientamenti politici del nuovo regime. Il giorno stesso del golpe, infatti, venne costituita la Giunta di governo, con a capo Augusto Pinochet e composta dai comandanti in capo dei tre rami delle Forze Armate e dal generale dei Carabinieri, che si attribuì il potere costituente, quello legislativo e quello esecutivo (Decreto Ley n. 1). Alcuni giorni dopo venne chiuso il Congresso nazionale, mentre a meno di un mese dalla presa del potere gli uomini in divisa dichiararono i partiti facenti parte della coalizione Unidad Popular «asociaciones ilícitas» (e ne disposero lo scioglimento e la confisca dei

10 Si veda anche El Mercurio: "Un año de construcción”, cit., pp. 21-23. 
beni, mentre il Partido Demócrata Cristiano ed il Partido Naciónal vennero dichiarati «en receso»). Molto rapidamente furono adottate anche misure finalizzate a sottomettere le autorità locali al controllo dei militari (sindaci e responsabili delle municipalità vennero sostituiti da soggetti scelti dal nuovo regime tra gli ufficiali in ritiro e personalità locali), e si riorganizzò tutto il settore pubblico, da cui vennero destituiti per ragioni politiche tutti i funzionari appartenenti o vicini alle formazioni della sinistra. In breve, «los militares tomaron el control del país con gran rapidez y sentaron las bases de un nuevo orden político, centralizando la autoridad en la Junta de Gobierno, que se convirtió en el principal órgano decisorio» (Huneeus, 2016: 120).

Altrettanto celere fu il controllo sui mezzi di informazione e comunicazione, che il regime conseguì chiamando in causa la sicurezza dello Stato e la necessità di garantire l'ordine ${ }^{11}$. Tutti i giornali di sinistra o, comunque, vicini alle forze che avevano fatto parte di Unidad Popular, vennero chiusi ed i rispettivi beni furono confiscati, mentre venne introdotta una stretta censura su tutta la stampa autorizzata a circolare. Stessa sorte toccò alla televisione e alle emittenti radio del paese.

Praticamente in contemporanea il regime mise in piedi un efficiente apparato con funzioni di propaganda costituito da organismi appositi che videro la partecipazione determinante di giornalisti, esperti di comunicazione, sociologi, psicologi. Struttura in cui, almeno in una prima fase, una posizione centrale venne occupata dalla Secretaría general de gobierno $^{12}$, che aveva alle proprie dipendenze organismi come la Dirección nacional de comunicación social (DINACOS) - le cui competenze andavano dal controllo sul rispetto delle norme sulla censura all'attività di disinformazione mediante una propaganda attiva -, la Dirección de relaciones humanas - che, fra le varie cose, contribuì all'elaborazione delle operazioni di "guerra psicologica" nel campo dell'informazione -, o il Comité creativo de asesoría publicitaria - le cui mansioni includevano la progettazione di campagne dirette a orientare l'opinione pubblica ${ }^{13}$.

L'azione della Giunta militare fu guidata, inoltre, sin dal principio, da una fondamentale componente di antimarxismo, che si sarebbe immediatamente convertito in uno

11 Junta De Gobierno De Las Fuerza Armadas Y Carabineros De Chile, Santiago: "Bando n. 5", 11/09/1973. Fin dall'11 settembre, infatti, la Giunta operò anche attraverso l'emissione di bandi, non riconosciuti dalla tradizione costituzionale cilena come fonte del diritto e, tra le leggi vigenti in tutto il periodo precedente al colpo di Stato, presenti solo all'interno del Codigo de Justicia Militar e nella Ley de Seguridad Interior del Estado, in precetti la cui costituzionalità non è mai stata unanimemente accettata (Garretón et al., 1998: 20).

12 A partire dal 1976 la Secretaría general de gobierno venne trasformata in ministero e divenne il principale organismo dedito alla mobilitazione del sostegno politico al regime.

13 Dalla metà del 1974, però, anche questa struttura subì alcune modificazioni di rilievo, che inclusero la sottoposizione dei suddetti organismi alle direttive della Dirección unica de informaciones (che rispondeva direttamente al capo di Stato), mentre la Secretaría general de gobierno venne chiamata ad occuparsi dell'attività di coordinamento dei ministeri e della gestione delle segreterie nazionali $d e$ la juventud, de la mujer e de los gremios. Sul ruolo di queste ultime nell'ambito della mobilitazione controllata del sostegno politico al regime si veda, in particolare, Huneeus (2016: 342-363). 
degli elementi cardine del discorso del nuovo regime, che contraddistinse anche i primi atti istituzionali di questo, ne orientò l'azione repressiva (e non solo) e fu marcatamente presente nella narrazione operata dai mezzi di informazione per almeno il primo anno di dittatura. Del resto, come aveva affermato il generale Gustavo Leigh la notte stessa dell'11 settembre, la missione che si erano date le FF.AA. era quella di extirpar el cáncer marxista, e questa sarebbe stata portata avanti fino alle estreme conseguenze ${ }^{14}$. Non a caso, il citato Bando n. 5 dell' 11 settembre del 1973, che costituì una sorta di estensione del menzionato Decreto Ley n. 1 con il quale era stata istituita la Junta de gobierno, venne quasi interamente dedicato al deposto governo Allende, bollato come illegittimo, contrario alla morale, non rappresentativo del sentire nazionale e responsabile di aver spezzato l'unità stessa della nazione. Le FF.AA., pertanto, avevano assunto il dovere morale, imposto dalla patria, di prendere il potere per il lasso di tempo richiesto dalle circostanze ${ }^{15}$.

Secondo Carlos Huneeus, la repressione applicata nel corso dei primi anni di dittatura non sarebbe da attribuirsi all'impatto della Dottrina della sicurezza nazionale (DSN), quanto, piuttosto, alle stesse linee di comportamento dei militari cileni, che, ben prima della seconda guerra mondiale, si erano caratterizzati per un certo anticomunismo. A suo giudizio, infatti, tre furono le decisioni fondamentali che avrebbero impresso un marchio altamente coercitivo alla dittatura: la decisione del comandante in capo della FACH, Gustavo Leigh, di bombardare durante il colpo di Stato il palazzo presidenziale con aerei dell'istituzione da lui presieduta, nonché il suo discorso in cui definiva la guerra al marxismo come una delle massime priorità del nuovo regime; la decisione del comandante in capo dell'Esercito, il generale Pinochet, di creare un servizio di sicurezza destinato a dirigere la guerra al marxismo - la Dirección de Inteligencia Nacional (DINA), con a capo Manuel Contreras ${ }^{16}$; e, infine, l'operazione condotta dal generale Sergio Arellano Stark finalizzata a rendere effettivo l'ordine del jefe de la nación di accelerare i processi dei tribunali militari nel nord del paese (in seguito alla quale vennero assassinate 72 persone, in quella che sarebbe passata alla storia come «la caravana de la muerte») ${ }^{17}$. A ciò sarebbe andata ad aggiungersi la notevole predisposizione di Pinochet nei confronti del ricorso alla violenza, concepita anche come una maniera di cancellare la sua stessa immagine di ultimo arrivato sul carro dei golpisti, visto che il comandante in capo dell'Esercito non aveva preso parte sin dall'inizio alla cospirazione (ivi: 120-121). Secondo Huneeus, infatti, i militari cileni non assunsero la Dottrina della sicurezza nazionale nel corso degli anni Sessanta, a differenza di altri eserciti latinoamericani. Solo dopo il colpo di Stato, mediante la collaborazione con i militari brasiliani, venne introdotta tale dottrina all'interno delle FF.AA. del Cile (ivi: 188-189).

14 Discorso del generale Gustavo Leigh dell'11 settembre 1973 (Huidobro, 1989: 263).

15 Junta De Gobierno De Las Fuerza Armadas Y Carabineros De Chile: "Bando n. 5", cit.

16 Su questo tema si vedano, tra gli altri: Dorat Guerra e Weibel Barahona (2012), Policzer (2014), Rebolledo (2013), Salazar (2011 e 2012).

17 Su questo tema si veda, tra gli altri, Verdugo (2006). 
Questa posizione non è condivisa dall'autore del presente articolo. La DSN apparve per la prima volta in Brasile, all'interno della "Escola superior de guerra", attraverso le teorizzazioni del generale Golbery do Couto e Silva (do Couto e Silva, 1955 e 1967), ma andò incontro a una notevole diffusione nelle accademie militari di diversi paesi latinoamericani ${ }^{18}$. Essa non fu, tuttavia, il mero prodotto dell' azione di indottrinamento condotta dalle Forze Armate statunitensi all'interno delle scuole militari latinoamericane, né ebbe a che vedere esclusivamente con la repressione in senso stretto realizzata dai regimi civico-militari del Cono Sud in funzione anti-sovversiva. La DSN rappresentò il risultato di un'integrazione progressiva di contributi interni ed esterni ai singoli paesi, di apporti militari e civili, che condussero le Forze Armate nazionali ad elaborare delle proprie specifiche versioni (A. Varas Et Al., 1980: 103). Un percorso che portò in diversi casi ad una trasformazione del tradizionale rapporto fra politica e uomini in divisa, aprendo le porte ad un intervento diretto di questi ultimi nella gestione dello Stato, reinterpretato come fenomeno "biogeografico", in cui sviluppo e sopravvivenza venivano considerati i principali istinti.

Nella prospettiva della DSN il mondo era diviso in due campi contrapposti e irriducibili, l'Occidente e il comunismo, e questo antagonismo doveva rappresentare il primo postulato non solo di politica estera, ma anche di politica interna di tutti gli Stati. Di questi due raggruppamenti contrapposti, l'uno rappresentava il bene e l'altro il male, e le nazioni non avevano altra scelta per la loro sopravvivenza se non quella di appartenere all'uno o all' altro ${ }^{19}$. La nazione era, infatti, vista come un organismo vivente all'interno del quale ogni singola parte era funzionale all'esistenza del tutto, dotato di un proprio ciclo vitale di nascita, crescita e morte e la cui funzione principale doveva essere quella di rafforzare il potere nazionale e di difendersi da ogni aggressione esterna ed interna (Varas, 1988: 100). Le caratteristiche fondamentali di questa nazione che andava difesa dal «nemico totalitario» e quelle della civiltà all'interno della quale si voleva che questa restasse, si condensavano nella nozione di Occidente cristiano, «in nome del quale questi regimi intesero legittimarsi» (Zanatta, 2010: 179). Nozione che conduceva a due risultati immediati: il primo era che la nazione da difendere era, appunto, «un organismo dotato di un'essenza, la cristianità, e votato all'unità nell'Occidente»; il secondo era che «un nemico», che aveva un nome ben preciso ma che si manifestava in modo sfocato, attentava all'una e all'altra (ivi: 181-182). Sulla base di tutto ciò, sostituendo le frontiere territoriali con quelle ideologiche e affiancando al nemico esterno quello interno, la Dottrina della sicurezza nazionale elaborava, quindi, il concetto di «guerra totale», ossia una guerra che: a) si sarebbe dovuta concludere con la disfatta completa e definitiva dello sconfitto; b) si sarebbe dovuta combattere a livello politico, economico, sociale, psicologico e solo in ultima istanza militare; c) non avrebbe fatto distinzioni tra periodi di pace e periodi di con-

18 Si veda in proposito anche Dinges (2004).

19 Si veda in propósito Comblin (1979). 
flitto; d) si sarebbe dovuta svolgere senza tempo, avrebbe dovuto, cioè, considerare quella marxista una minaccia permanente (Arriagada Herrera, 1979: 177-180). Una guerra che, per la capacità stessa della "sovversione comunista" di infiltrarsi in maniera subdola e perversa in tutti i meandri della società, non solo avrebbe avuto ad oggetto un nemico indeterminato, ma avrebbe rappresentato una lotta per il controllo della popolazione, una guerra di carattere soprattutto politico-psicologico, il cui obiettivo era, cioè, la conquista fisica e psicologica delle popolazioni (ivi: 173-174).

La doctrina de la seguridad nacional se presenta como una síntesis total de todas las ciencias humanas, una síntesis dinámica capaz de proporcionar un programa completo de acción en todas las áreas de la vida social; una síntesis entre política, economía, ciencias psicosociales, estrategia militar. Ella se propone determinar los criterios definitivos en todas las áreas de la acción desde el desarrollo económico hasta la educación o la religión. En el mundo moderno sólo el marxismo tuvo una pretensión semejante a la ciencia total y la conducción total de la sociedad ${ }^{20}$.

In Cile, se è vero che, sul piano della sistematizzazione dottrinaria, fu con la $D e$ claración de principios del marzo del 1974 che, come ha sostenuto Genaro Arriagada, «la doctrina de la guerra contrasubversiva y elementos del pensamiento geopolítico, que se ubican en el centro de una versión específica de la doctrina de seguridad nacional», vennero fuori «en un rol significativo», è possibile sostenere che sin dal principio della dittatura questi elementi furono presenti nel discorso del regime (Arriagada Herrera, 1985: 76).

Quindi, d'accordo con Verónica Valdivia, nel caso del Cile: «El anticomunismo explícito de Leigh y de los otros miembros de la Junta, reflejado en todos los bandos del día del golpe, junto a los afanes de transformación presentes en los discursos de todos los altos oficiales eran parte de un mismo fenómeno: la centralidad de la seguridad nacional» (Valdivia, 2010: 167). Infatti, se nel corso degli anni Sessanta le tesi della "guerra contro-insurrezionale" e la stessa Dottrina della sicurezza nazionale furono lette dalle Forze Armate cilene come un qualcosa di "lontano", pur esercitando su di esse un certo influsso - e ciò anche alla luce del fatto che in Cile, a differenza di altri contesti, come quello argentino, non era presente una minaccia armata rivoluzionaria reale -, la situazione cambiò durante il periodo di Unidad Popular; al punto che, verso la fine del governo di Allende « se fue produciendo, entre importantes segmentos de los altos mandos de las tres ramas - aunque no de todos sus comandantes en jefe -, una interpretación común de la realidad, en el marco de la seguridad nacional» (ivi: 169).

È in questo quadro, quindi, che è possibile comprendere perché i militari cileni ritennero centrale il ricorso agli strumenti tipici della Guerra de propaganda y de ner-

20 Mensaje, Santiago: P. J. Comblin, "La Doctrina de la Seguridad Nacional”, 247, mayo-abril 1976, p. 96, citato in Tapia Valdés (1980: 104). 
vios fin da subito, attraverso tutti gli strumenti a disposizione (atti istituzionali, radio, televisione, organi di stampa, libri, opuscoli e così via): «la guerra solo podría ser ganada con la misma fe profesada por el enemigo, haciendo uso de idénticas técnicas de guerra psicológica, de propaganda y oponiendo una alternativa ideológica: la guerra contrasubversiva era la guerra total» (ivi: 168).

Oggi, peraltro, sembrano esservi sempre maggiori elementi che permettono di sostenere che i militari cileni fossero tutt'altro che degli sprovveduti sul fronte della propaganda e della "guerra psicologica"21. Anni dopo il ritorno alla democrazia in Cile, ad esempio, l'ex portavoce della Giunta militare, Federico Willoughby-MacDonald, avrebbe ammesso che il famigerato Plan $Z$ - il presunto complotto che Allende e le formazioni di UP sarebbero stati in procinto di mettere in campo con il fine di realizzare una sorta di auto-golpe per assumere il controllo totale del paese $-{ }^{22}$, altro non fu che «una maniobra de la guerra sicológica de los servicios de inteligencia que estaban entrenados para eso y que definieron que era conveniente crear algunos elemento de justificación del pronunciamiento militar [...] para convencer a la población de que ellos los habían salvado» ${ }^{23}$.

21 Su questo tema si vedano tra gli altri: Molina-Vedia (1976-77); Oquendo-Villar (2008); Timmermann (2014). Sul carattere consapevolmente simbolico di alcune azioni intraprese dai militari sin dal momento del golpe al fine di «explicitar el dominio y el ejercicio del poder», si veda, invece, il lavoro di Errázuriz e Leiva Quijada (2012). Di particolare interesse, in tal senso, è anche il reportage giornalistico realizzato da Jorge Escalante per il quotidiano cileno La Nación, relativo a una serie di documenti ritrovati all'interno de La Moneda al principio degli anni Duemila, che hanno dimostrato come, sin dalle prime ore del colpo di Stato, le alte sfere del regime fecero ricorso ad uno specifico gruppo di esperti, riuniti nel Dipartimento di psicologia della Direzione di relazioni umane guidato dallo psicologo Hernán Tuane e posto alle dipendenze della Secretaría general de gobierno, per condurre operazioni di guerra psicologica (Escalante, 2002: 3-4).

22 Il "mito del Plan Z" venne costruito sulle pagine de El Mercurio, che per settimane pubblicò le "prove" del suddetto complotto. Queste sarebbero poi state raccolte e sistematizzate all'interno del Libro blanco de la Junta militar de gobierno, preparato dagli organismi della propaganda del regime con il contributo di due collaboratori della CIA (U.S. Senate, 1975: 44). Al suo interno, oltre alla descrizione dettagliata del piano, si tentò di dimostrare come Salvador Allende non avesse mai goduto del sostegno della maggioranza dei cileni e come il governo di Unidad Popular fosse andato avanti violando ripetutamente le leggi dello Stato, tra cui la stessa Costituzione, e compiendo azioni criminali e ruberie (Secretaría General De Gobierno De Chile, 1973). Sul ruolo attivo de El Mercurio nell'edificazione del "mito del Plan Z' e, più in generale, nella propaganda del regime, si vedano, tra gli altri: Lagos (2009); Guzmán (2015: 181-226).

23 Huismann e Sohr (2003). Affermazioni che sempre Willoughby avrebbe ribadito circa dieci anni dopo, nel 2013, in occasione di un'intervista rilasciata al quotidiano cileno The Clinic: «Yo tengo la impresión que la gente encargada de las operaciones de inteligencia discernieron que era conveniente generar un elemento de justificación del pronunciamiento militar para convencer a la población civil que los habían salvado. Entonces se hizo este libro y se produjo incluso un efecto social [...] Este libro [Libro blanco del cambio de gobierno en Chile, in cui venne esposta la tesi del Plan Z] le repito, es producto de una campaña de guerra sicológica» (The Clinic online, Santiago: "Secretario de Prensa de la Junta Militar sobre el Plan Z: «Fue una gran maniobra de guerra sicológica»", 2/09/2013 - http://www.theclinic. cl/2013/09/02/el-plan-z-la-mentira-que-ensangrento-a-chile/). Sempre Willoughby avrebbe sostenuto, peraltro, che, al fine di evitare episodi di resistenza, già nelle ore del colpo di Stato, i militari decisero di infondere «miedo, mucho miedo a la población» (Willoughby-MacDonald, 2012: 153). Willoughby, fu secretario de prensa y vocero della Junta militar, carica che avrebbe mantenuto per quasi due anni 
In breve, l'estirpazione del "cancro marxista" andò ben oltre la repressione in senso stretto. Essa si avvalse di una massiccia propaganda, delle tecniche della guerra psicologica e, come hanno raccontato nel loro golpe estético Luis Hernán Errázuriz e Gonzalo Leiva Quijada, intervenne pesantemente sulla quotidianità dei cileni, modificando (limpiando) e decontestualizzando ogni campo, dall' ambito culturale nel senso più ampio del termine ${ }^{24}$ alla «percepción y configuración estético-social de la ciudad» (Hernán Errázuriz e Leiva Quijada, 2012: 8). E, proprio perché la guerra dichiarata al marxismo non puntava solo alla sconfitta politica di questo, ma mirava alla sua demolizione a livello ideologico, alla sua cancellazione completa e definitiva in ogni settore, essa richiedeva, appunto, strategie che andassero oltre la repressione, strategie che in qualche modo chiamassero in causa anche il concetto stesso di consenso. Non a caso, «el régimen, lejos de restringir sus vínculos única y exclusivamente a los núcleos empresariales, fue a la captura de exponentes de los sectores populares, asociados en la época a la izquierda y el centro político» (Valdivia, 2010: 166).

Nella prima fase della dittatura tutto partecipò alla creazione di questa immagine di paese in cui governo e Forze Armate e di polizia stavano conducendo una guerra (totale) contro un nemico che aveva praticamente invaso il territorio nazionale; un clima da guerra interna utile a giustificare la violenza da parte del regime (non motivata dalla presenza di una concreta minaccia sovversiva ${ }^{25}$, a rendere accettabile agli occhi della popolazione la sua azione repressiva e a creare consenso intorno all'operato della Giunta. Una situazione funzionale a mantenere elevata la tensione e la paura nella popolazione - una risposta di vera e propria «confusión y miedo», come è stato affermato (Lira Kornfeld e Castillo, 1991: 125-127) - o, per essere più precisi, a suscitare a comando determinate emozioni e sentimenti a seconda delle necessità. Remarono in tale direzione la retorica ufficiale degli uomini del regime, ma anche i suoi atti istituzionali, fra cui i bandi - «El lenguaje se hace bando ${ }^{26}$ asumiendo una modalidad perentoria de amenaza» (ivi: 130) -, tra le prime comunicazioni ad essere manipolate ai fini di quella che è stata definita la

e mezzo, fino al febbraio del 1976, al fine, come racconta lui stesso nella sua biografia, di «unificar criterios y dar a conocer hacia afuera, frente al país, una sola información, para dar la visión de unidad» (ivi: 159-160).

24 Si veda in proposito anche Brunner (1981).

25 In Cile, dalla metà degli anni Sessanta, era presente il Movimiento de Izquierda Revolucionaria (MIR), che era fautore della conquista del potere attraverso la lotta armata (su nascita ed evoluzione dell'organizzazione in questione fino al 1970, si veda: Palieraki, 2014). Tuttavia, il MIR non fu mai un gruppo guerrigliero nel senso pieno del termine (Valdivia, 2010: 168), e, soprattutto, neanche questo riuscì ad esercitare una qualche forma di opposizione militare al regime nell'immediato post-golpe (Winn, 2013: 123 e ss.). Più in generale, l'azione delle Forze Armate non incontrò quasi alcuna forma di resistenza, anche in città tradizionalmente di sinistra come Concepción, la terza del paese per importanza, caratterizzata dalla presenza di una grande zona industriale, di sindacati combattivi e di un certo peso e di una altrettanto rilevante università in cui lo stesso Movimiento de Izquierda Revolucionaria esercitava una notevole influenza (Huneeus, 2016: 114-115). 
«producción discursiva de miedo» (Timmermann, 2014: 140-141) ${ }^{27}$. Ma operarono in tal senso soprattutto i mezzi di informazione, a cominciare dagli organi di stampa, che non si limitarono alla creazione del menzionato "mito" del Plan Z, ma seguitarono per mesi nell'azione di screditamento delle forze politiche del precedente esecutivo ${ }^{28}$; e, soprattutto nei mesi successivi al colpo di Stato, agendo da strumento di diffusione di teorie, idee e notizie provenienti dal cuore della dittatura, fecero in modo che quella comunista, ogni qual volta reputato necessario, venisse percepita come una minaccia concreta e attuale ${ }^{29}$. Agevolando, così, il compito della Giunta militare nell'ambito della propria auto-legittimazione e sul piano della conduzione della guerra sul fronte interno. E ciò, molto spesso, in applicazione di vere e proprie operazioni di guerra psicologica elaborate dagli esperti del regime ${ }^{30}$.

27 Bandi che nel loro complesso assolsero alla duplice funzione di dar vita ad un clima «emocional psicosocial» tale da rendere molto più vulnerabile e manipolabile la popolazione e di svolgere un'attività informativa che fu sempre propagandistica, non pretendendo «dar cuenta de una realidad sino interpretarla» (Garretón et al., 1998: 19).

28 Sulle pagine del quotidiano El Mercurio, ad esempio, alla stregua di un romanzo d'appendice a puntate, vennero ricostruite tutte le principali tappe del cammino intrapreso dal precedente governo, nell'ambito di una Breve historia de la Unidad Popular (ribattezzata, ben presto, Breve Historia del Régimen Marxista), il cui fine fu quello di denigrare pesantemente il precedente esecutivo. Una ricostruzione accompagnata da singoli articoli diretti a mettere in evidenza la contraddizione fra gli ideali di cui tali forze si erano fatte promotrici ed il supposto "dietro le quinte" svelato dai militari, ovvero, come suggerivano gli esperti della guerra psicologica della Giunta, «el contraste entre lo publicitario de la UP y su realidad interna"» (Escalante, 2002: 4). Una "realtà" fatta, quindi, di ruberie (El Mercurio: "Sólo en Valparaíso se Buscan 312 Vehículos Fiscales Desaparecidos", 2/10/1973, p. 21), protezione di traffici illeciti come quello della droga (El Mercurio: "Por 30 mil dólares al mes. La UP Protegía el Tráfico de Drogas", 17/10/1973, p. 1), conti privati depositati in banche all'estero (El Mercurio: "Dirigentes "UP" Tenían Cuentas Privadas en Bancos de Londres", 26/09/1973, p. 15), utilizzo della cosa pubblica per affari privati (El Mercurio: "Hombres de Negocios en la Intimidad de UP", 3/10/1973, p. 19; El Mercurio: "Allende y Castro Tenían Consorcio de Negocios", 14/10/1973, p. 33), sperpero di denaro pubblico (El Mercurio: "Caótico Estado de Hacienda Pública", 26/09/1973, p. 15; El Mercurio: "250 Mil Impresos Marxistas Costaron E 10.000.000", 9/10/1973, p. 18; El Mercurio: "Propaganda Marxista", 11/10/1973, p. 3), corruzione (El Mercurio: "JAPS: Centros de Fraudes Económicas Y Actos Terroristas", 30/09/1973, p. 33; El Mercurio: "9 Detenidos en Escándalo De los Autos 'Mapu-125”, 5/10/1973, p. 17), politiche fallimentari in ambito economico e sociale (El Mercurio: "Dejaba Sin Alimentos a Escolares: Politizada y Desorganizada la Junta de Auxilio", 28/09/1973, p. 17; El Mercurio: "La UP Empobreció A los Chilenos", 10/10/1973, p. 15; El Mercurio: "Ruina Total del Cobre Causó Régimen Marxista", 1/10/1973, p. 2; El Mercurio: "UP Tenía Evidencia De la Crisis del País", 16/11/1973, p. 21) che avevano finito per spianare la strada alla penetrazione della "sovversione" nel paese (El Mercurio: "Infiltración Del Castrismo En Chile", 5/11/1973, p. 1).

29 Sarebbe impossibile menzionare tutti gli articoli che, secondo che scrive, rientrano in questo tipo di discorso. Si vedano, solo a titolo di esempio: El Mercurio: "Marxismo Protege a Delincuentes", 18/01/1974, p.18; El Mercurio: "Reviven Tácticas Y Métodos Marxistas", 22/01/1974, p. 3; El Mercurio: "El Comunismo en Reorganización", 30/01/1974, p. 2; La Tercera de la Hora, Santiago: “«Hay por lo menos cinco arsenales en Santiago»", 6/02/1974, p. 3; Las Últimas Noticias: "Marxistas Están Internando Armas", 20/02/1974, p. 27; La Segunda, Santiago: "La médula del antimarxismo", 16/03/1974, p. 3.

30 Ad esempio, nel novembre del 1973, un dossier intitolato Preparación sicológica de la población para contrarrestar la acción marxista venne inviato a Pinochet e agli uomini della Giunta. Si trattava di un 
Ma la propaganda e la "guerra psicologica" della dittatura sul fronte interno non riguardarono unicamente i danni prodotti dal precedente esecutivo e la lotta alla sovversione. Anche se questi aspetti non vennero mai meno, parallelamente a questo, a conferma del fatto che il regime operò inizialmente all'interno dei parametri "ideologici" della Dottrina della sicurezza nazionale, si affermò una narrazione che vedeva nei militari l'ultimo baluardo a difesa dello spirito e dell'identità stessa della nazione, messi sotto attacco dalla penetrazione di ideologie "straniere". Era a loro che, pertanto, spettava il compito di proteggere l'unità della patria, recuperandone virtù e simboli tradizionali.

In breve, il regime civico-militare dimostrò sin dal primo momento di essere perfettamente consapevole del fatto che nessuna dittatura si reggeva facendo esclusivamente ricorso alla violenza; ma, soprattutto, come accennato, era impegnato in una guerra totale che mirava alla «derrota definitiva del marxismo» (Valdivia, 2010: 199). Anche per questo l'antimarxismo che, come detto, monopolizzò l'azione e la narrazione del regime autoritario cileno e di tutti i suoi apparati nel corso del primo anno di gestione nel potere, venne accompagnato da tentativi di mobilitazione della popolazione intorno agli obiettivi nazionali fissati dalla Giunta di governo e da strategie finalizzate ad ottenere il consenso di questa. Fu sicuramente questo il caso della mobilitazione controllata di juventud, mujer e gremios - ovvero, alcune delle categorie principali del movimiento cívico culminato con il colpo di Stato del 1973 e che non a caso vennero organizzate in segreterie nazionali sotto la direzione della Secretaría general de gobierno -, che furono considerate fondamentali per «obtener una masa ciudadana organizada para el apoyo de la Junta cuando sea necesario» ${ }^{31}$. Ma rientrarono in questo ambito anche operazioni e campagne ad hoc come: Comprométase con CHILE, con la quale la Giunta, già a pochi giorni dal golpe,

piano di guerra psicologica preparato dal Departamento de sicología de la Dirección de relaciones humanas posto alle dipendenze della Secretaría general de gobierno. Al suo interno si manifestava la preoccupazione che, una volta svanito «"el temor paralizante de la acción bélica"», la sinistra potesse riorganizzarsi e tentare di influenzare l'opinione pubblica; per questo motivo occorreva agire «"a semejanza de lo realizado en días posteriores al 11 de septiembre en la Guerra Sicológica, donde se dejó en evidencia el contraste entre lo publicitario de la UP y su realidad interna"». Per fare ciò sarebbe stato necessario «"preparar sicológicamente a la población”», in primo luogo attraverso una «"lucha contra el movimiento UP en general"», creando «"la obtención de estos vínculos"»: «"Marxismo (UP) equivalente a Mentira"», «"Marxismo (UP) igual Traición”», «"Marxismo (UP) igual Corrupción”»; $\mathrm{e}$, in secondo luogo, mediante «"una campaña contra el extremismo"»: «"Extremismo igual AntiCileno"», «"Extremismo igual Mercenarios”», «"Extremismo igual Inseguridad”», «"Extremismo igual Peligro"», «"Extremismo igual Pérdida de Libertad”». Tutto ciò sarebbe dovuto avvenire mediante la somministrazione di immagini semplici ed il ricorso a frasi brevi e idee chiare, esattamente come era stato fatto nei giorni successivi all'11 settembre con la «"Guerra Sicológica”» (Escalante, 2002: 3-4).

31 Come precisò il secretario general de gobierno al principio del 1974. Actas de la Honorable Junta de Gobierno (d'ora in avanti AHJg), n. 72, Santiago de Chile, 17 de Enero de 1974, p. 6; in Biblioteca del Congreso Nacional de Chile (d'ora in avanti, BCN). Sulla nascita, compiti e funzioni delle segreterie nazionali della gioventù, delle donne e dei gremios si veda Huneeus (2016: 342-363). Sull'importanza attribuita dalla dittatura alla juventud si veda, in particolare, González (2015), mentre sulla strumentalizzazione della figura e del ruolo de la mujer sin dagli anni dell'opposizione al governo Allende si guardi Power (2008). 
invitò la popolazione a contribuire alla ripresa del paese donando gioielli ed effetti personali di valore ${ }^{32}$ (ottenendo in cambio una targa o un anello senza alcuna importanza economica ma dal grande significato simbolico) ${ }^{33}$; o El Desafío, pensata dalla Secretaría general de gobierno con il contributo del suo Comité creativo ${ }^{34}$ e finalizzata a compattare la popolazione intorno alla missione del governo in un momento di difficoltà economica del paese ${ }^{35}$.

In pratica, come emerse nel corso di una delle consuete riunioni segrete dei membri della Giunta militare, tenutasi al principio del 1974, alla presenza del cuore stesso del settore comunicazione del regime, ovvero di personaggi come Pedro Ewing (capo della Secretaría general de gobierno), Gastón Acuña (Director de informaciones de gobierno) e il noto giornalista Alvaro Puga - riunione in cui si discusse della necessità di una maggiore centralizzazione dell'attività di comunicazione e informazione dinanzi al pericolo di una perdita di consenso intorno all'azione del governo - si reputava necessario sradicare le cause stesse che avevano dato origine all'allendismo attraverso un «un cambio profundo en la mentalidad del país» ${ }^{36}$.

32 AhJg, n. 5, 19 de Septiembre de 1973, p. 2; in BCN.

33 Ahjg, n. 8, 24 de Septiembre de 1973, p. 1; in BCN. Per diverso tempo circolò sulle pagine di tutti i giornali un manifesto con l'invito «Comprométase con la Patria, llevando un aporte, cualquiera que sea, a todos los Bancos del país. Contribuya a la restauración nacional, ayudando a financiar a CHILE», scritta accompagnata dal disegno di una mano intenta nell'inserire un anello all'anulare sinistro di un'altra mano, collegata alla quale vi era una bandiera cilena.

34 AHJg, n. 93, 14 de Febrero de 1974, p. 2; in BCN.

35 È possibile che essa fu elaborata con il contributo degli esperti di guerra psicologica del regime. Un memorandum preparato dal Departamento de sicología de la Dirección de relaciones humanas della Secretaría del gobierno per il segretario generale di quest'ultima, infatti, presumibilmente tra la fine del '73 e l'inizio de '74, e intitolato Chile de pie frente al desafío de la crisis económica mundial, offre diverse indicazioni in tal censo. All'interno del documento venivano segnalati quelli che erano gli obiettivi da raggiungere nell'ambito della comunicazione da portare avanti per contrastare gli effetti sull'opinione pubblica della difficile situazione economica attraversata dal paese. E questi obiettivi erano: «a) Focalizar la atención de la población en acontecimientos AJENOS al acontecer nacional. b) Realizar un trabajo psicológico subliminal, a modo tal, que la ciudadanía lige el problema internacional como causa de la crisis económica interna». La seconda fase avrebbe avuto, a sua volta, tre obiettivi: «Ligar situación internacional y sus consecuencias en países subdesarrollados mencionando a Chile»; «Recordar como análisis de final de año el PASADO U.P: y sus SIGNIFICACION COMO DESTRUCCION»; «LIGAR SUBLIMINALMENTE DOS ACONTECIMIENTOS NEGATIVOS PARA CHILE», ovvero «CRISIS MUNDIAL» e «ACCION DESTRUCTORA U.P.». La quarta fase avrebbe avuto, poi, due obiettivi principali, legati assieme: «a) Crear sentimiento de solidaridad, y que todos comparten sacrificio [...] b) Frente a la situación de guerra interna, se debe enfrentar a los enemigos». Chi erano i nemici in questione? «1) Extremista: que corre rumores falsos habiendo efectivamente sacrificios compartidos. 2) Enemigo económico: el que se niega a compartir sacrificios y aprovecha para sacar ventajas: el especulador y el acaparador. 3) Enemigo social: el que se niega a trabajar a toda su capacidad. 4) Indiferente: el que no se confonde con el desafío» (Archivo Nacional de Chile, Ministerio de Educación, Oficios reservados recibidos, $\mathrm{n}^{\circ} 23$, p. 1 e ss.). Su questi aspetti si veda anche: Guida, 2020.

36 A tal proposito Gastón Acuña sottolineava l'importanza di: dar corso al progetto di decreto-legge che avrebbe dato vita al ministero della Secretaría general de gobierno, individuare nuovi mezzi di comunicazione che permettessero di mantenere il contatto con la cittadinanza, favorire una mobilitazione di massa imponente a favore dei piani e dei progetti del governo - a tale scopo, la Secretaría general non 


\section{1974: VERSO UNA NUOVA STRATEGIA COMUNICATIVA}

Tuttavia, alla metà del 1974, a quasi un anno dalla presa del potere, la Giunta militare seguitava a definire sé stessa prevalentemente per contrapposizione con il deposto regime. E ciò sia sul fronte "interno" che su quello "esterno" 37 . In realtà, proprio quest'ultimo rendeva sempre più evidente la necessità di un cambio nella strategia comunicativa del regime. Sul piano internazionale, infatti, il caso cileno aveva cessato di essere unicamente l'oggetto delle proteste di una parte (importante) dell'opinione pubblica internazionale, di qualche governo e dell'attenzione dei media, per divenire ufficialmente motivo di dibattito all'interno di organizzazioni come quella degli Stati Americani o le Nazioni Unite. Con la Risoluzione 3219 sulla "Protezione dei diritti umani in Cile", ad esempio - che aveva visto l'astensione degli Stati Uniti - nel novembre del '74 l'Assemblea generale dell'ONU aveva espresso profonda preoccupazione «relating to gross and massive violations of human rights» che si stavano producendo nel paese in questione ${ }^{38}$. E al principio del 1975 la Commissione per i diritti umani delle Nazioni Unite avrebbe approvato, con la Risoluzione $\mathrm{N}^{\circ} 8$ (XXXI), la costituzione di un Gruppo di lavoro ad hoc che investigasse da vicino sul problema della violazione dei diritti umani e delle libertà fondamentali in Cile ${ }^{39}$. Con tutte le conseguenze che ciò comportava per l'immagine internazionale del paese latinoamericano, sempre più isolato sul piano diplomatico ed economico. Non a caso Jaime Guzmán ${ }^{40}$ - considerato uno dei principali ideologi del regime -, alcuni giorni prima che si tenessero le celebrazioni per il primo anno dal «pronunciamiento» dell'11 settembre, aveva affermato che queste avrebbero dovuto rappresentare la risposta «masiva» del Cile al mondo, finalizzata a dimostrare che la maggioranza del popolo sentiva come suo questo primo anniversario e guardava a questa data come all'inizio della ricostruzione nazionale:

avrebbe potuto continuare ad essere un mero organismo di coordinamento, ma avrebbe dovuto fungere da strumento attraverso il quale la Giunta potesse dirigere una vera e propria azione politica (AHJG, $\mathrm{n}$. 73, 18 de Enero de 1974, pp. 1-3; in BCN).

37 Basti pensare che Il Libro blanco del cambio de gobierno en Chile rappresentava ancora la principale pubblicazione inviata in giro per il mondo e distribuita attraverso le varie ambasciate cilene, accompagnata da testi come Anatomía de un fracaso. La experiencia socialista chilena, Chile 70-73, crónica de una experiencia (entrambi degli autori Hernán Millas ed Emilio Filippi), Gestión económica del Gobierno de la Unidad Popular (di Alberto Baltra), ovvero scritti propagandistici che puntavano essenzialmente a mettere in evidenza i danni prodotti dal deposto governo Allende e, pertanto, a giustificare l'intervento delle Forze Armate.

38 United Nations-General Assembly (1974).

39 «The resolution was tabled by Senegal (combining drafts provided by the UK, Netherlands, Nicaragua and the USSR) and was adopted without a vote. The Ad Hoc Working Group on Chile would be composed of five experts and was to report to the Commission at its 32 nd session. Four years later, the Ad Hoc Working Group was transformed (partly to reduce costs) into a Special Rapporteur on the situation of human rights in Chile, the first Special Rapporteur with a country mandate» (Limon e Power, 2014: 6).

40 Su Jaime Guzmán si vedano, tra gli altri, Cristi (2011), Gazmuri (2013), Moncada Durruti (2006). Non è possibile, per ragioni di spazio, approfondire in questa sede il suo ruolo in materia di propaganda. Ad ogni modo, stando alle fonti consultate, non è possibile al momento attribuirgli la responsabilità della definizione di linee di indirizzo. 
«Los chilenos debemos mostrar al mundo que somos un país liberado, lejos de ser oprimido, y que la reconstrucción detrás de la cual actúa la unidad de todo el pueblo, está encabezada por nuestros legítimos gobernantes [...] En este sentido - agregó - celebro que el acto no vaya a tener una connotación política al estilo tradicional, desde el momento en que no habrá oradores que pretendan arrogarse la representación de la civilidad, sino que será el pueblo entero el que reunido en sus organizaciones irá a asumir su compromiso con Chile» ${ }^{41}$.

Inoltre, approssimativamente nello stesso periodo, stava maturando nel cuore della dittatura una svolta programmatica reale, una sterzata che andava nella direzione di un nuovo progetto-paese, un proyecto fundacional destinato a rompere drasticamente con il passato, a cominciare proprio dall'ambito economico. Tanto è stato scritto sull'importanza dei cosiddetti Chicago Boys per la dittatura cilena. La loro collaborazione con la dittatura iniziò molto presto, poco dopo il colpo di Stato, quando Fernando Léniz venne designato ministro dell'Economia e portò con sé, come collaboratore, Sergio de Castro ${ }^{42}$ (Huneeus, 2016: 383). Ad ogni modo, la maggior parte degli studiosi è concorde nel ritenere che, al momento del golpe, i militari non contavano su di un progetto economico condiviso, ma che, anzi, vi fosse una prevalenza fra gli esponenti delle FF.AA. di coloro i quali ritenevano necessarie riforme sociali ed economiche di carattere strutturale (Valdivia, 2001). In pratica, anche se, fra il 1973 ed il 1975, iniziarono ad essere adottate anche misure di stampo liberista e politiche di desestatización, la tendenza prevalente nella Giunta era ancora quella tradizionale, nacional-desarrollista, gradualista, che mirava, prima di ogni altra cosa, alla stabilizzazione economica ed al contenimento dell'inflazione (Gárate Chateau, 2012: 188). Fu il perdurare dell'instabilità monetaria, che si aggravò nel corso del 1974, a facilitare la penetrazione della linea "estrema" dei tecnici di Chicago, convinti sostenitori di una drastica riduzione del debito pubblico, del peso dello Stato nell'economia e dell'apertura indiscriminata del paese al commercio estero (ivi: 189). Fu così che, a partire dal 1975, iniziò la fase «de implantación ideológica del modelo neoliberal», con il Plan de Recuperación Económica, meglio conosciuto come "trattamento shock" (ivi:197). Gli ufficiali desarrollisti vennero definitivamente messi da parte, Pinochet consolidò definitivamente la propria leadership all'interno del regime, e il paese imboccò quella strada che avrebbe condotto al cosiddetto "miracolo economico cileno" (1977-1981).

Tutto ciò è importante anche perché la gestione economica non fu neutrale, ma era ovviamente strettamente legata alla strategia di legittimazione dell'ordine autoritario (Huneeus, 2016: 365-366); sia sul piano interno che su quello esterno. A livello interno,

41 El Mercurio: “«Acto en el Parque Bustamante Será la Respuesta de Chile al Mundo»”, 8/09/1974, p. 4.

42 Quest'ultimo era stato tra i realizzatori de El Ladrillo, un programma di riforme economiche di stampo neoliberista - ma non di carattere monetarista - a cui avevano lavorato, a partire dal 1972, economisti dell'Universidad Católica e dell'Universidad de Chile su richiesta della Marina, allo scopo di preparare un programma economico di emergenza nell'eventualità di un colpo di Stato (Huneeus, 2016: 382-383 e 721-722). 
ad esempio, sarebbe stato impossibile gestire gli effetti della crisi economica e, successivamente, le ripercussioni di misure shock come quelle contemplate dal Plan de Recuperación Económica ricorrendo unicamente alla repressione. Ma, più in là di questo, come ha scritto Verónica Valdivia:

la nueva mentalidad chilena, a la que desde un principio aspiró el régimen militar, no habría de surgir solo de la represión o de la economía neoliberal, sino sería producto también de un trabajo sistemático de reeducación cívica desarrollado por organismos oficialistas, cuya tarea sería generar un nuevo sistema de valores que constituiría el nuevo consenso social [...] La Secretaría General de Gobierno fue el instrumento usado por el régimen para difundir y legitimar las medidas neoliberales adoptadas [...] Televisión Nacional de Chile, como medio oficialista y dependiente de lo que sería DINACOS, fue utilizada para justificar dicha política [...] A su vez, las Secretarías utilizaron al voluntariado que cobijaban - tanto mujeres como jóvenes - para difundir los nuevos ideales sociales de la austeridad económica, el ahorro, la lucha contra la inflación y el repliegue socioeconómico del Estado [...] Las voluntarias se diseminaron por los campamentos y poblaciones del país para explicar a las mujeres pobres la economía del consumidor, la importancia de administrar los escasos bienes familiares como si fuesen una empresa, y el respaldo que requería el régimen para reconstruir un país destruido por el estatismo y la demagogia, mientras los jóvenes invitaban a su generación a una «cruzada de solidaridad» [...] Una vez que el gobierno se decidió por una política económica de corte monetarista, su éxito dependía no solo de la represión - los años más tenebrosos de la DINA -, sino de difundir las nuevas verdades precisamente entre los más afectados por el desempleo, la caída de los salarios y el repliegue de la ayuda del Estado ${ }^{43}$. (Valdivia, 2012: 33-35).

Al di fuori della più ampia azione propagandistica cui si è accennato in precedenza, finalizzata, fra le varie cose, a radicare l'idea che i problemi economici del paese fossero il prodotto delle devastazioni compiute dal precedente governo e, in subordine, della crisi internazionale, e a compattare la popolazione intorno alle "sfide" di volta in volta individuate dalla Giunta e nell' ambito della lotta che questa stava conducendo contro un nemico subdolo che attentava all'esistenza stessa della nazione, vennero adottati progressivamente dei piani specifici per tenere "a bada" la popolazione ${ }^{44}$. Eppure, ciò non servì a placare le preoccupazioni del regime sul fronte del consenso interno.

43 Verónica Valdivia Ortiz de Zárate, La alcaldización de la política, Los municipios en la dictadura pinochetista, LOM, Santiago de Chile, 2012, pp. 33-35.

44 Tra questi, ad esempio, un programma di diffusione permanente organizzato dalla Secretaría General attraverso il quale, ogni martedì, sulla Televisione nazionale venivano spiegate le ragioni della crisi e le misure che stava adottando il governo per superarla; conferenze tenute in differenti comunità locali; trasmissioni radio costanti mediante le quali, appunto, si cercava di propagandare il concetto che le difficoltà che si stavano incontrando fossero essenzialmente il prodotto di un problema globale. Tuttavia lo stesso Pinochet sottolineò la necessità di condurre una campagna ancora più ampia e approfondita, a tappeto, población per población, per propagandare le conquiste ottenute dal governo in ambito economico (Si veda in proposito: AHJg, n. 171-A, 14 de Noviembre de 1974, pp. 1-11). 
Esattamente in questa fase, quindi, in particolar modo il personale civile vicino al governo militare iniziò a ritenere che sul fronte della propaganda, sia all'interno che al di fuori del paese, fosse giunta l'ora di cambiare registro. Di fondamentale importanza furono una serie di incontri tenutisi alla metà del 1974 tra i membri della Giunta e alcuni esperti di comunicazione provenienti dall' esperienza imprenditoriale, i quali arrivarono a sottolineare la necessità che in questo settore si ricorresse ad un nuovo tipo di approccio, molto diverso da quello che era prevalso nei mesi precedenti. Come emerse nel corso della lunga riunione segreta del 23 luglio di quell'anno, ad esempio, il cambio si sarebbe dovuto produrre in maniera graduale, senza brusche alterazioni che fossero andate a stravolgere ciò che era stato fatto sino allora ed evitando, al contempo, di confondere un «público» che si era ormai abituato a ricevere un certo tipo di immagini; ciò nondimeno, esso avrebbe dovuto essere radicale ${ }^{45}$.

Fino a quel momento, tutta l'immagine della Giunta si era basata, infatti, «en una política de contraste». Questo non era ritenuto più sufficiente. Non bastava dichiararsi critici di quello che c'era stato prima, né affermare di essere la "patria restaurata". La Giunta avrebbe dovuto incarnare la creazione di cose vitali e positive, la costruzione di una nuova società, di un «nuevo Chile» ${ }^{46}$.

Tuttavia, come J.F. Kennedy non aveva partorito dalla sera alla mattina l'idea dell'Alleanza per il progresso, come lo "spazio vitale" di Hitler era stato il prodotto del lavoro di filosofi tedeschi che avevano riflettuto a lungo per individuare qualcosa che potesse servire «de título o de cuño» per questa esperienza, allo stesso modo, una trasformazione di questo tipo, secondo gli esperti del regime, non si sarebbe potuta realizzare da un giorno all'altro, né si sarebbe potuta lanciare con un editoriale de El Mercurio o con un'intervista rilasciata a Las Últimas Noticias o a La Tercera. Questa «nueva imagen» avrebbe dovuto costituire una pietra miliare, «un hito rodeado de muchas cosas y preparado de antemano», il cui annuncio, realizzato facendo leva anche su di una certa spettacolarità, avrebbe dovuto produrre un reale contrasto con il passato, incluso con il proprio passato:

Entonces, creemos que el 11 de septiembre es la fecha clave, es la fecha en que debiera ser el lanzamiento de todo, el lanzamiento del cuño, la exteriorización del Presidente de la Junta de las virtudes de la Junta; una exposición sobre el pasado, lo que se ha hecho en el intertanto, y el lanzamiento del nuevo Chile con gran dramatismo, con gran espectacularidad ${ }^{47}$.

Sino ad allora, sarebbe stato necessario insinuare alcune cose tra la popolazione, ma, esattamente come a teatro prima che si alzi il sipario, stando bene attenti a non svelare

45 AhJg, n. 138, 23 de Julio de 1974, p. 10; in BCN. Dal verbale della riunione in questione non è possibile stabilire con certezza l'identità di questi esperti provenienti dal mondo imprenditoriale.

46 Ivi, p. 3.

47 Ibidem. 
troppo, a non rivelare il segreto, causando in questo modo la perdita dell'impatto, dell'effetto del «golpe de contraste».

La cerimonia dell' 11 settembre del 1974 avrebbe dovuto rappresentare, quindi, il momento chiave, il grande palcoscenico da cui lanciare la nuova "idea-forza", ossia, «la Junta, el Presidente de la Junta y este nuevo Chile». «Todo lo interesante, todo lo medular, todo lo que tiene substancia va a venir el 11», e la popolazione avrebbe dovuto ricevere delle novità, avrebbe dovuto sentirsi fortemente colpita da queste persone che stavano conducendo il paese verso nuovi traguardi e nuovi orizzonti.

Ciò sarebbe stato realizzato attraverso un grande discorso pronunciato dal generale Pinochet, fatto di analisi, definizione dei traguardi raggiunti e di proiezioni verso il futuro. Ma i concetti chiave, questo passato, questo presente e questo futuro avrebbero richiesto, a loro volta, di essere tradotti all'interno di ogni singolo settore, in campo economico, in quello sociale, in quello agricolo, e così via. Ciascun tema trattato avrebbe dovuto contenere «una nota atractiva y nueva para el público para simbolizarle esta nueva sociedad, este nuevo Chile que se inicia». In economia, ad esempio, sarebbe dovuto emergere il nuovo spirito dinamico, quello che veniva fuori dalla rinnovata importanza conferita alla pesca, al mare, al krill, quest'ultimo presentabile addirittura come un potenziale sostituto del rame in termini di ricchezza per la nuova nazione ${ }^{48}$; o, ancora, le possibilità offerte dall'esportazione del vino, la cui rilevanza non era mai stata compresa prima di allora. Nel settore dell' agricoltura, invece, si sarebbe dovuto evidenziare il passaggio da una produzione di sussistenza ad un'economia che funzionava per sfamare non una sola famiglia, ma tutto il paese, mettendo fortemente in risalto, anche qui, il contrasto con il passato ${ }^{49}$.

Più in generale, occorreva presentare una nuova mappa del Cile, una nuova concezione, un nuovo oggetto geopolitico, qualcosa a cui la gioventù potesse aggrapparsi, che mostrasse come l'Europa fosse ormai alle spalle e il futuro andasse ricercato in altre aree e in altri obiettivi ${ }^{50}$. Ma tutto questo non sarebbe valso a nulla se tutte le alte sfere del regime non si fossero trasformate in grandi direttori d'orchestra, «en Wagner», dando vita a qualcosa di realmente spettacolare, tale da lasciare il pubblico a bocca aperta e da produrre un impatto anche all'estero. Nulla, in pratica, nell'organizzazione dell'once, si sarebbe dovuto lasciare al caso.

Inoltre, questi enormi sforzi non avrebbero condotto da nessuna parte senza «lo que los ingleses llaman el "follow-up"», ossia «la continuación, la persistencia»" ${ }^{51}$ Per questi motivi, sottolineavano gli esperti, lo «psicólogo y sociólogo Sr. Tuane» (a capo del Departamento de sicología de la Dirección de relaciones humanas) e «todos los demás» che si stavano occupando della propaganda, si sarebbero dovuti dimenticare, dopo lo spartiacque rappresentato dall'11 settembre del 1974, di tutti quei «programitas» in cui

\begin{tabular}{ll}
\hline 48 & Ivi, p. 5. \\
49 & Ivi, p. 7 \\
50 & Ivi, p. 6. \\
51 & Ivi, p. 8.
\end{tabular}


erano coinvolti, per adattarsi una volta per tutte a questa nuova visione geopolitica del Cile e per portare avanti queste nuove idee e questi nuovi concetti:

\begin{abstract}
Naturalmente, los publicistas, los relacionadores públicos y los propagandistas tienen que hacerle todo un calendario, toda una serie de proposiciones para realizar lo que Ud. diga, en el país y en el extranjero. Esto no puede desvincularse de conceptos que se den a las Embajadas. El Ministro de RR. EE. que anda por aquí en relación a la imagen pública, tiene que integrarse, porque los conceptos que se digan en todos estos discursos tienen que procesarse, tanto para Ud., tanto para Ud., y esto tiene que sistematizarse. Y al Ministro de RR. EE. le van a tocar una serie de actividades, porque tiene que informar a las Embajadas, subrayar los puntos importantes, dar instrucciones a los embajadores de que pesquisen periodistas amigos y los instruyan, en fin, toda una serie de relaciones públicas necesarias. Y si no lo hacemos, vamos a perder el 11 de septiembre, y ya nos hemos puesto muy cicateros en este empleo y en esta optimización de recursos. El 11 de septiembre es para nosotros una inversión muy grande. Si no lo aprovechamos, estamos haciendo una mala inversión ${ }^{52}$.
\end{abstract}

E non solo il ministro Ismael Huerta, ma tutti i responsabili dei ministeri ed i loro collaboratori avrebbero dovuto tradurre queste nozioni, entrando loro stessi a far parte della squadra che si sarebbe occupata di questo, al pari dei giornalisti, sia a livello nazionale che internazionale; perché «eso es lo que, a la larga, nos va a cambiar la imagen en el extranjero, y eso es lo que nos va a afianzar la buena imagen interna» ${ }^{53}$.

Entonces, en síntesis [...] creemos nosotros que, como en todo teatro - y esto es teatro; así es la política: es teatro -, los grandes actores y los únicos actores son Uds [...] Y en este teatro hay una aparición de los actores, dramática, impactante, el 11 de septiembre, fecha simbólica, histórica. Para eso preparémonos, pero no caigamos en el error de que vamos a borrar las calles que llevan a ese teatro principal; aprovechemos las calles existentes, pero pavimentémoslas, pongamos alguna alfombra. Eso lo debe hacer la propaganda actual readaptada por nosotros ${ }^{54}$.

A tale scopo, quindi, era necessario che venissero definite il prima possibile le "coordinate", la mappa vera e propria di questo nuovo Cile, pensando anche ad un simbolo fisico capace di rappresentare questo «nuevo país» che i militari stavano costruendo e che pensavano di realizzare ${ }^{55}$. Ma occorreva anche che loro stessi fossero messi al corrente, e nei minimi dettagli, delle campagne di propaganda in marcia sino a quel momento - le quali non si ponevano gli obiettivi di cui si stava parlando -, perché era esattamente così che funzionava nel mondo della pubblicità organizzata a livello imprenditoriale:

\begin{tabular}{ll}
\hline 52 & Ivi, pp. 8-9. \\
53 & Ivi, p. 9. \\
54 & Ivi, p. 10. \\
55 & Ivi, p. 11.
\end{tabular}


Por ejemplo, si deseo una campaña para vender un producto y el publicista me dice: Ya, lo voy a hacer así y asá; ésa es la primera conversación. Pero la segunda conversación ya se hace con los planos, los mapas, los dibujos, la firma y el texto, y no se lanza la campaña hasta que no está absolutamente de acuerdo con las comas y los puntos. Y eso, en las campañas políticas, es la misma cosa; porque un productor de éstos que no tiene por qué saber más, puede terminar con los más altos ideales por un error tonto ${ }^{56}$.

Nel campo della propaganda esistevano, infatti, in quel momento, due grandi organismi esecutivi, la Dirección Nacional de Comunicación Social (DINACOS), guidata dal colonnello Virgilio Espinoza ${ }^{57}$, e l'Asesor Cultural de Gobierno. Quest'ultimo avrebbe dovuto rappresentare, secondo costoro, una sorta di «embajador blanco», colui che «vende la imagen de la Junta con una tarjeta impecable que es la cultura ${ }^{58}$. Si trattava, infatti, di una «paloma blanca, insospechada», l'unica in grado di introdurre «ideas de tipo socio-político, de guerra psicológica, etc., con esa catadura: la Cultura, con C mayúscula». A questa figura sarebbe toccato un posto di primo piano nel processo di «cocientización del país», attraverso l'assunzione di funzioni specifiche come quella di «relacionador público del Gobierno», di creatore del «nuevo vocabulario chileno, sustitutivo del marxista», di responsabile dell' «adoctrinamiento de Chile, para llenar el universo cultural chileno, que estaba inundado con la letrina de "Clarín" y de las demás porquerías, con nuevos símbolos» ${ }^{59}$. Intorno all'Asesor Cultural sarebbe dovuto ruotare il vero indottrinamento del paese attraverso i nuovi concetti, approfittando di tutto quel che offriva la situazione, come «la ausencia de oposición» e il fatto di poter contare su di una figura «que se puede intercalar ahí sin despertar ninguna sospecha, como es lo cultural». Non si trattava, però, di divinizzare un uomo oppure di caricarlo di eccessive attribuzioni; il responsabile della cultura, al pari di tutti gli altri, era solo uno strumento, che serviva nella misura in cui e fino a quando costituiva un buon strumento ${ }^{60}$.

Quanto al responsabile della DINACOS, Virgilio Espinoza, il giudizio sul suo operato era positivo: anche se c'erano alcune cose che mancavano e altre ancora che andavano corrette, questi stava lavorando bene. Eppure, ribadivano gli esperti, occorreva voltare

$56 \quad$ Ivi, p. 10.

57 Ufficiale di Stato Maggiore, professore militare ed esperto di intelligence, il colonnello Espinoza si era disimpegnato precedentemente, fra le varie cose, come Director de Operaciones del Estado Mayor General del Ejército, Secretario de Estudios de la Escuela de Infanteria, Segundo Comandante del Regimiento "Tucapel" a Temuco e Comandante del Regimiento "Pudeto" a Punta Arenas. Espinoza aveva seguito anche dei corsi negli Stati Uniti sulla "Difesa Continentale" presso il Collegio Interamericano di Difesa di Washington (La Patria, Santiago: "Dispuso el Gobierno: Mayor agilidad en labores informativas", 27/06/1974, p. 9).

58 AHJG, n. 138, cit., p. 11. Il riferimento era con ogni probabilità a Enrique Campos Menéndez, noto giornalista e scrittore che, nel dicembre del 1974, sarebbe stato ufficialmente nominato Asesor Cultural de la Honorable Junta de Gobierno.

59 AhJg, n. 138, cit., p. 11.

60 Ibidem. 
pagina, sebbene senza immediati stravolgimenti, «porque ya la gente se habituó a eso, pero cambiando el tono y llevándolo a esto que se ha hecho» ${ }^{61}$.

Dalla riunione segreta del 23 luglio, quindi, veniva fuori l'ampia convergenza degli astanti su di un punto fondamentale: i metodi utilizzati sino ad allora e, più in generale, il vecchio modo di fare propaganda, non potevano essere più considerati adeguati a fronteggiare le sfide che si paravano dinanzi alla Giunta:

Había una campaña desastrosa, que es la de disco. El psicólogo y el sociólogo nos anunciaron que estaban por hacer algo que se llama Los Principios de la Junta, en canciones. El primer lado serían canciones con los Principios de la Junta, que aparecerían subliminalmente.

El señor Presidente de la Junta: Serían como canciones de iglesia.

El señor Almirante Merino: Como las que tenía la Unidad Popular.

$\mathrm{Y}$ al dorso, en la otra cara del disco, salían pensamientos. Entonces, estamos en las mejores tradiciones de Mao. Es una ridiculez. A mí me dio un estremecimiento.

El señor General Leigh: A mí me trajeron las carpetas de las campañas que están apareciendo en El Mercurio. Me mencionaron que había 4 más ${ }^{62}$.

«El fondo» era stato ben definito. Fino all'11 settembre si sarebbe ricorso alla propaganda di sempre, con poche variazioni. Da quel momento in avanti, invece, con la benedizione dei membri della Junta de gobierno, si sarebbe voltato pagina, adattando «inmediatamente el follow-up, la propaganda posterior» ai nuovi concetti ${ }^{63}$.

\section{CONCLUSIONI}

L'impiego sistematico della violenza rappresentò, indubbiamente, un fattore centrale, un elemento che contraddistinse il regime autoritario cileno. Del tutto eccezionali e senza ritorno, si potrebbe dire, anche per il panorama latinoamericano, furono, infatti, il carattere apertamente sanguinario della Giunta militare e la rottura del sistema politico, sociale ed economico vigente cui il nuovo regime avrebbe dato luogo. L'istituzionalizzazione di un vero e proprio "Stato del terrore", mediante il ricorso metodico ai più diversi metodi repressivi, rappresentò un tratto tipico della dittatura cilena. Tuttavia, quest'ultima non governò esclusivamente attraverso la violenza fisica. La stessa "ideologia della sicurezza nazionale" partiva dal presupposto che quella contro il comunismo fosse una lotta per il controllo della popolazione, una guerra di carattere soprattutto politico-psicologico, il cui obiettivo era, cioè, la conquista fisica e psicologica delle popolazioni.

\begin{tabular}{ll}
\hline 61 & Ibidem. \\
62 & Ivi, p. 19. \\
63 & Ivi, p. 20.
\end{tabular}


Per tali motivi, visto che in gioco vi era la conquista dei "cuori e delle menti" dei cileni, due dei punti cardine della DSN non potevano che essere la propaganda e la "guerra psicologica". Inizialmente, queste si fondarono soprattutto sulla legittimità controrivoluzionaria e anticomunista, concentrando il loro messaggio sulla diffamazione del governo Allende e di Unidad Popular e facendo di quella "sovversiva" una minaccia pressoché permanente. Parallelamente a questo, prevalsero, oltre a una narrazione che descriveva l'intervento dei militari come il prodotto della responsabilità patriottica dell'istituzione custode dello spirito della nazione, il richiamo costante a concetti e valori quali l'unità della nazione, la patria, la moralità, la necessità di ripristinare virtù e simboli tradizionali dell' "idiosincrasia" cilena, nonché il tentativo di compattare e di mobilitare, anche, la popolazione intorno agli "obiettivi nazionali” della Giunta di governo. E, sia nella retorica del regime che nella narrazione operata dai mezzi di informazione, l'associazione fra extremismo, foráneo, antinacional, antipatriótico, e così via, si fece sempre più netta ed incalzante, anche a conferma di come nell'ambito della guerra anti-sovversiva fosse crollata ogni barriera tra fronte interno ed esterno.

Tuttavia, verso la metà del 1974 iniziò a manifestarsi con forza la necessità di un cambio di rotta nella strategia comunicativa. La "retorica antisovversiva" non sembrò più essere sufficiente a garantirsi un certo consenso sul fronte interno e a limpiar l'immagine del regime su quello internazionale. La propaganda avrebbe dovuto cambiare tono, messaggio e strumenti: lasciando da parte l'anticomunismo (anche se mai del tutto), bisognava sorprendere i cileni e il mondo intero vendendo «una cosa nueva», che non era più semplicemente opposizione al passato, né, tantomeno, riproduzione, ricostruzione o ricostituzione; ma, piuttosto, costruzione di «una nueva sociedad», di un «nuevo Chile».

Gli effetti di questo mutamento di direzione si sarebbero visti solo nel corso degli anni successivi, a cominciare in particolare dal 1976 e, ancor di più, dal 1977, quando la macchina propagandistica della dittatura iniziò a contare su risorse, uomini e mezzi sempre più efficaci e, soprattutto, quando l'obiettivo principale divenne quello di favorire la diffusione della percezione del Cile in termini di paese assolutamente normale: una nazione pacificata, moderna, unita, che desiderava la pace e la tranquillità necessarie al suo sviluppo. 


\section{FONTI E BIBLIOGRAFIA}

\section{Archivi:}

Archivo Nacional de Chile-ministerio de Educación

Biblioteca del Congreso Nacional de Chile

Archivo general histórico del ministerio de Relaciones exteriores de Chile

\section{Volumi:}

Arriagada Herrera, G. (1979): El pensamiento político de los militares (Estudios sobre Chile, Argentina, Brasil y Uruguay), Santiago de Chile, CISEC.

Arriagada Herrera, G. (1985): La Política Militar de Pinochet, Santiago de Chile.

Brunner, J. (1981): La cultura autoritaria en Chile, Santiago de Chile, FLACSO.

Comblin, P. J. (1979): Dos ensayos sobre seguridad nacional, Santiago de Chile, Vicaría de la Solidaridad.

Cristi, R. (2011): El pensamiento político de Jaime Guzmán: autoridad y libertad, Santiago de Chile, LOM.

Dinges, J. (2004): The Condor Years: How Pinochet and his Allies Brought Terrorism to three Continents, New York, New Press.

Do Couto e Silva, G. (1967): Geopolítica do Brasíl, Río de Janeiro, José Olympio Editora.

Do Couto e Silva, G. (1955): Planejamento Estratégico, Río de Janeiro, Biblioteca do Exército, Vol. 213, Companhia Editora Americana.

Dorat Guerra, C., Weibel Barahona, M. (2012), Asociación ilícita. Los archivos secretos de la dictatura, Santiago de Chile, CEIBO.

Errázuriz, L. H., Leiva Quijada, G. (2012): El Golpe Estético. Dictadura militar en Chile 1973-1989, Santiago de Chile, Ocho Libros Editores.

Escalante, J. (2002): “Como la Dictadura hizo la guerra sicológica”, Santiago de Chile, La Nación.

Gárate Chateau, M. (2012): La revolución capitalista de Chile (1973-2003), Santiago de Chile, Universidad Alberto Hurtado.

Garretón, M. A. et al. (1998): Por la fuerza sin la razón. Análisis y textos de los bandos de la dictadura militar, Santiago de Chile, LOM.

Gazmuri, C. (2013): ¿Quién era Jaime Guzmán?, Santiago de Chile, RIL Editores.

González, Y. (2015): “El 'Golpe Generacional' y la Secretaría Nacional de la Juventud: Purga, disciplinamiento y resocialización de las identidades juveniles bajo Pinochet (1973-1980)", Atenea 512 (II), pp. 87-111.

Guida, A. (2020): "Dottrina della sicurezza nazionale, educazione e cultura nel Cile di Augusto Pinochet (1973-1975)", Roma, Processi Storici e Politiche di Pace-Rivista di Storia e Politica Internazionale, 23-24 (XII), 57-86. 
Guzmán, N. (2015): Los Agustines: el clan Edwards y la conspiración permanente, Santiago de Chile, CEIBO Ediciones.

Huidobro, S. (1989): Decisión naval, Valparaíso, Imprenta de la Armada.

Huismann, W., Sohr R. (2003): Pinochet Plan Z, documentario, Arte GEIE /WDR /Huismann

Huneeus, C. (2016): El Régimen de Pinochet, Santiago de Chile, Taurus.

Jara Hinojosa, I. (2011): "Graficar una "segunda independencia": el régimen militar chileno y las ilustraciones de la Editorial Nacional Gabriela Mistral (1973-1976)", Santiago De Chile, Historia, 44 (1), 131-163.

Junta De Gobierno De Las Fuerza Armadas Y Carabineros De Chile (1974): República de Chile 1974. Primer año de la reconstrucción nacional, Santiago de Chile, Editora Nacional Gabriela Mistral.

Lagos, C. (2009): El Diario de Agustín. Cinco estudios de casos sobre El Mercurio y los derechos humanos (1973-1990), Santiago de Chile, LOM.

Limon, M., Power, H. (2014): History of the United Nations Special Procedures Mechanism. Origins, Evolution and Reform, Universal Rights Group.

Lira Kornfeld, E., Castillo, M. I. (1991): Psicología de la amenaza política y el miedo, Santiago de Chile, Editorial ChileAmérica-CESOC.

Loveman, B. (1999): For la Patria: Politics and the Armed Forces in Latin America, Wilmington (DE), Scholarly Resources.

Molina-Vedia, S. (1976-77) "El Caso Chileno Como un Prototipo de Guerra Psicológica Contrarrevolucionaria", Ciudad de México, Revista Mexicana de Ciencias Políticas y Sociales.

Moncada Durruti, B. (2006): Jaime Guzmán. Una democracia contrarevolucionaria. El político de 1964 a 1980, Santiago de Chile, Ril Editores.

Oquendo-Villar, C. (2008): "Chile 1973 - El golpe y la voz de la ley”, E-misférica, (http:// hemisphericinstitute.org/journal/3.1/esp/es31_pg_oquendo_villar.html).

Pinochet Ugarte, A. et al. (1974): Un año de construcción. 11 Septiembre 1973-11 Septiembre 1974, Santiago de Chile, Talleres graficos del Servicio de Prisiones.

Palieraki, E. (2014): ¡La revolución ya viene!: El MIR chileno en los años sesenta, Santiago de Chile, LOM.

Policzer, P. (2014): Los modelos del horror. Represión e información en Chile bajo la Dictadura Militar, Santiago de Chile, LOM.

Power, M. (2008): La mujer de derecha. El poder feminino y la lucha contra Salvador Allende, 1964-1973, Santiago de Chile, DIBAM.

Rebolledo, J. (2013): El despertar de los cuervos. Tejas Verdes, el origen del exterminio en Chile, Santiago de Chile, Ceibo Ediciones.

Salazar, M. (2011): Las letras del horror. Tomo I: La DINA, Santiago de Chile, LOM.

Salazar, M. (2012): Las letras del horror. Tomo II: La CNI, Santiago de Chile, LOM.

Secretaría General De Gobierno De Chile (1973): Libro blanco del cambio de gobierno en Chile. 11 de Septiembre de 1973, Santiago de Chile, Lord Cochrane. 
Tapia Valdés, J. A. (1980): El terrorismo de Estado. La Doctrina de la Seguridad Nacional en el Cono Sur, Santiago de Chile, Editorial Nueva Imagen.

Timmermann, F. (2014): El Gran Terror. Miedo, Emoción y Discurso. Chile, 1973-1980, Santiago de Chile, Ediciones Copygraph.

U.S. Senate (1975): Covert Action in Chile: 1963-1973, Washington, U.S. Government Printing Office.

United Nations-General Assembly (1974): Protection of human rights in Chile, Res. $\mathrm{N}^{\circ}$ 3219 (XXIX), (http://www.un.org/documents/ga/res/29/ares29.htm).

Valdivia Ortiz De Zárate, V. (2010): “'Estamos en guerra, señores!'. El régimen militar de Pinochet y el 'pueblo', 1973-1980”, Santiago De Chile, Historia, I (43), 163-201.

Valdivia Ortiz de Zárate, V. (2001): "Estatismo y Neoliberalismo: un contrapunto militar. Chile 1973-1979”, Santiago de Chile, Historia, 34, 167-226.

Valdivia Ortiz de Zárate, V. (2012): La alcaldización de la política, Los municipios en la dictadura pinochetista, Santiago de Chile, LOM.

Varas, A. (1988): La politica de las Armas en America Latina, Santiago de Chile, FLACSO.

Varas, A. et al. (1980): Chile, Democracia, Fuerzas Armadas, Santiago de Chile, FLACSO.

Verdugo, P. (2006): Gli artigli del puma, Milano, Sperling \& Kupfer.

Willoughby-MacDonald, F. (2012): La Guerra. Historia íntima del poder en los últimos 55 años de política chilena 1957-2012, Santiago de Chile, Momentum.

Winn, P. (2013): La revolución chilena, Santiago de Chile, LOM.

Zanatta, L. (2010): Storia dell'America Latina contemporanea, Roma-Bari, Laterza. 